\title{
Correction to: Downregulation of fibronectin 1 attenuates ATRA-induced inhibition of cell migration and invasion in neuroblastoma cells
}

\author{
Xiaolin Tan ${ }^{1}$ - Wei Gong ${ }^{1} \cdot$ Bo Chen ${ }^{1} \cdot$ Baocheng Gong ${ }^{1} \cdot$ Zhongyan Hua $^{1}$. Simeng Zhang ${ }^{1} \cdot$ Yang Chen $^{1} \cdot$ Qi Li $^{1}$. \\ Zhijie Li ${ }^{1}$
}

Published online: 28 February 2022

(c) Springer Science+Business Media, LLC, part of Springer Nature 2022

\section{Correction to: \\ Molecular and Cellular Biochemistry \\ (2021) 476:3601-3612 \\ https://doi.org/10.1007/s11010-021-04113-5}

In the original publication of the article, the invasion image of FN1-siRNA-transfected SY5Y cells in Fig. 5B, the invasion image of ATRA-treated NGP cells in Fig. 7B and the

\section{Zhijie Li}

lizhijie68@hotmail.com; lizj@sj-hospital.org

1 Medical Research Center, Liaoning Key Laboratory of Research and Application of Animal Models for Environmental and Metabolic Diseases, Shengjing Hospital of China Medical University, \#36 Sanhao Street, Heping District, Shenyang 110004, China 
A

Left

Middle

Right
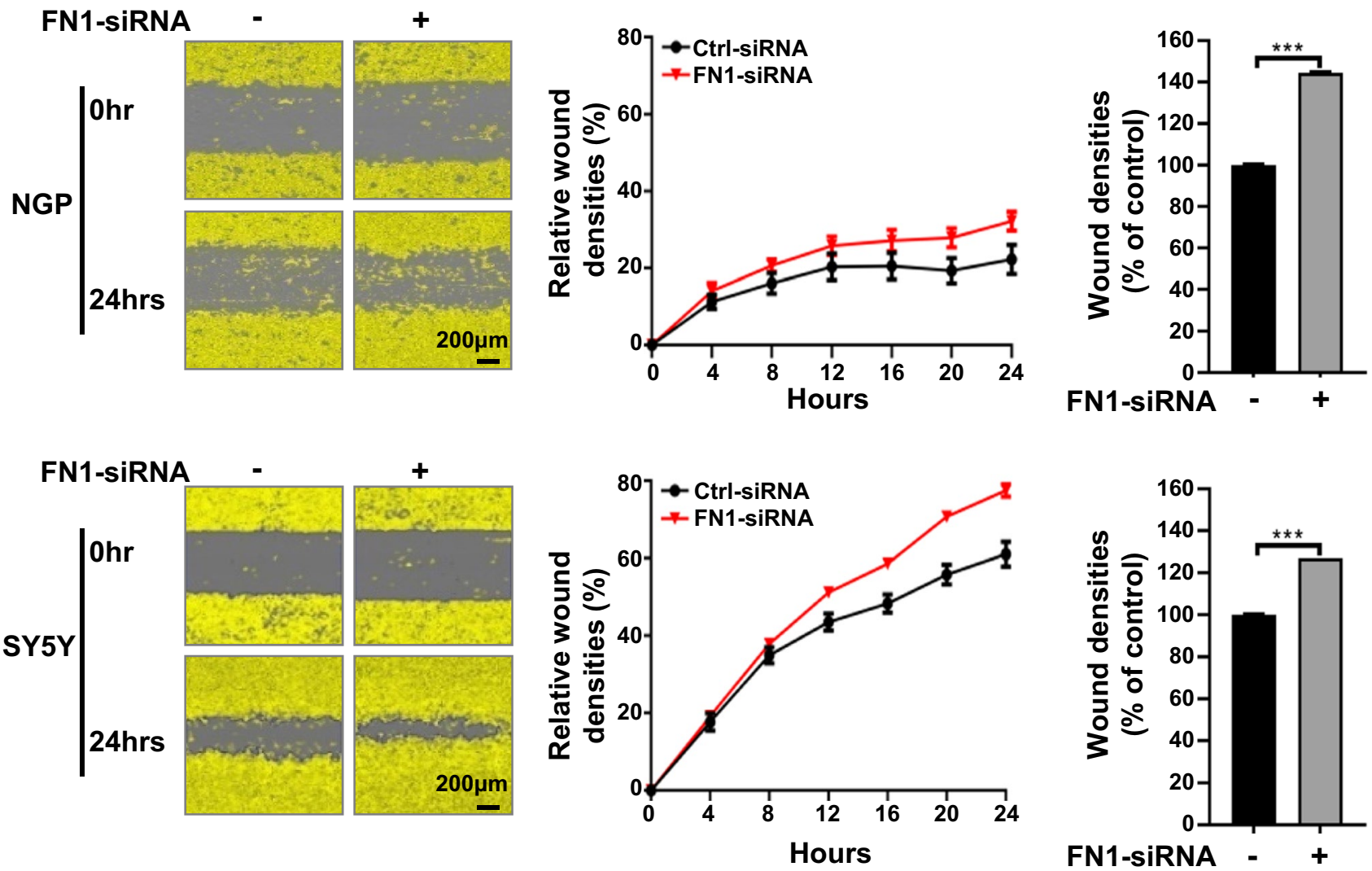

B
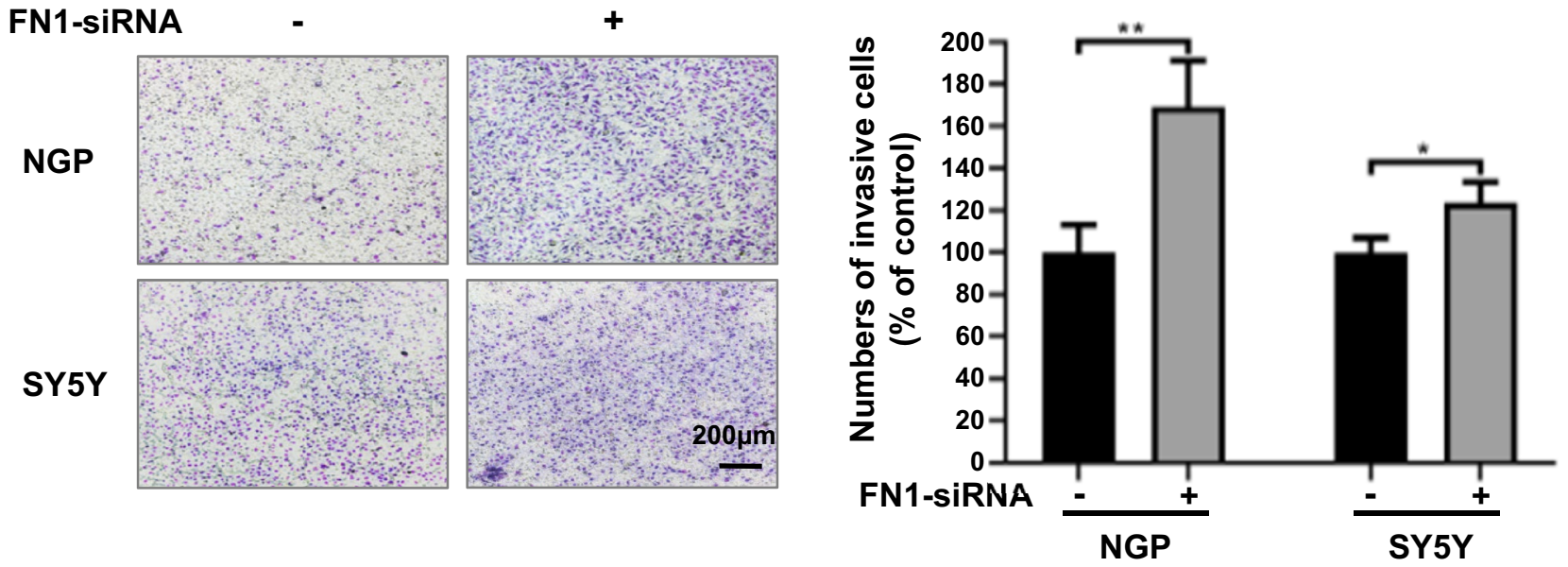

Fig. 5 Downregulation of FN1 increased migration and invasion of NB cells. A wound healing assay for two NB cell lines transfected with FN1-siRNA. Data were presented as representative microscopic images taken with an IncuCyte ZOOM (left), relative wound densities (middle) and relative wound densities at the $24 \mathrm{~h}$ time point (right).

B A transwell invasion assay for two NB cell lines transfected with FN1-siRNA. Data were presented as representative microscopic images (left) and relative numbers of invasive cells (right). ${ }^{*} P<0.05$; $* * P<0.01$. Scale bar $=200 \mu \mathrm{m}$ 
A

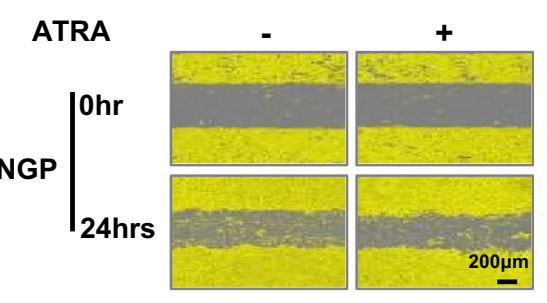

ATRA

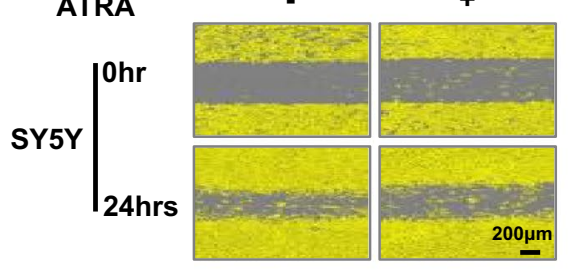

B

ATRA

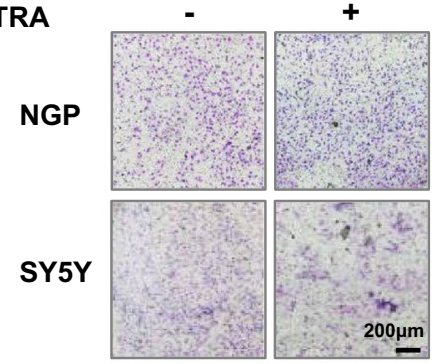

C

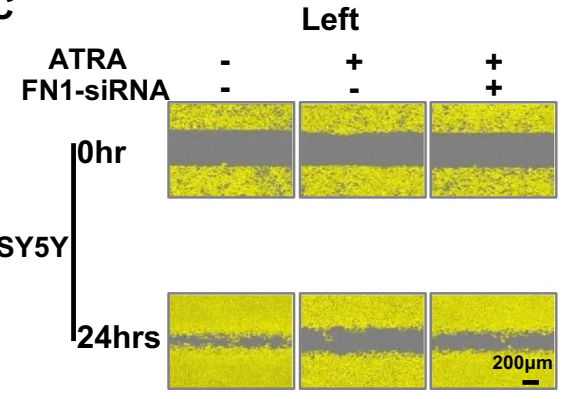

Middle
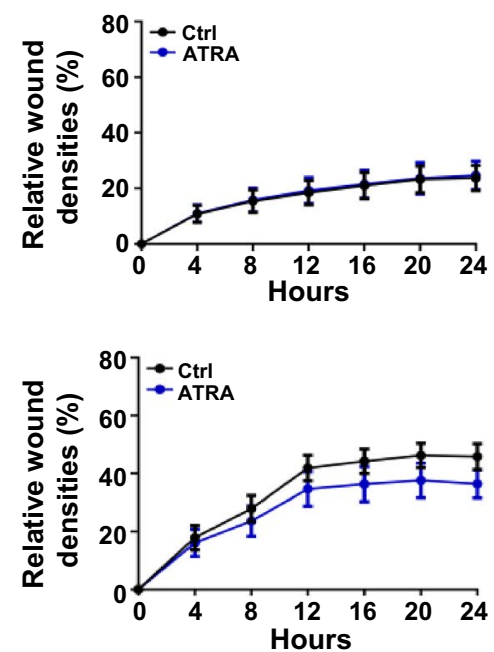

Right
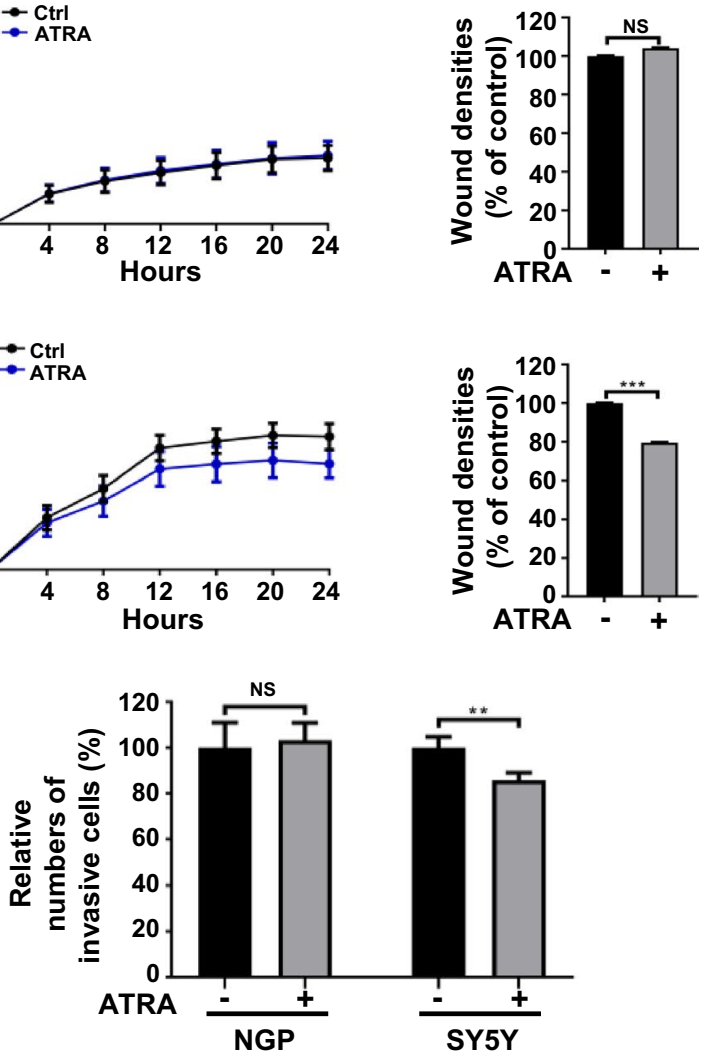

Middle

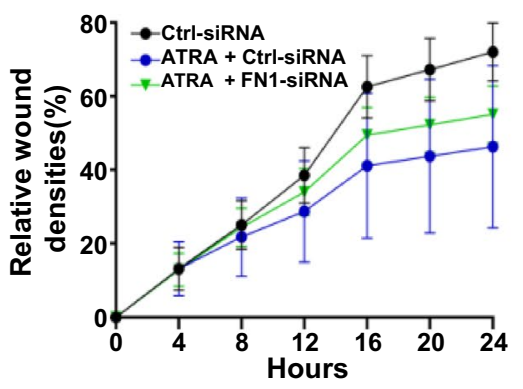

Right

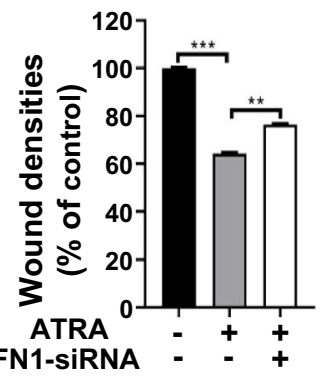

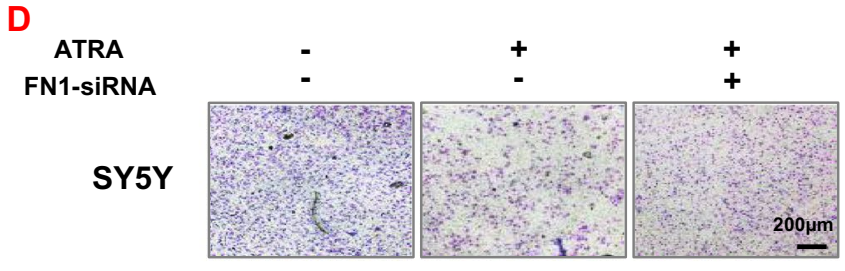

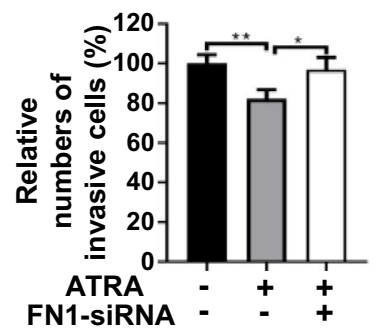

Fig. 7 Downregulation of FN1 partially blocked ATRA-mediated inhibition of cell migration and invasion in SY5Y cells. A Data from a wound healing assay, showing representative microscopic images (left), relative wound densities (middle) and relative wound densities at the $24 \mathrm{~h}$ time point (right). B Data from a transwell invasion assay, showing representative microscopic images (left) and relative numbers of invasive cells (right). C Data from a wound healing assay for SY5Y cells, showing rep- resentative microscopic images (left), dynamic changes of relative wound densities (middle) and relative wound densities at the $24 \mathrm{~h}$ time point (right). D Data from a transwell invasion assay for SY5Y cells, showing representative microscopic images (left) and relative numbers of invasive cells (right). NS no statistical significance. Scale bar $=200 \mu \mathrm{m}$ 
invasion image of control-siRNA-transfected SY5Y cells in Figure 7D were published incorrectly. The correct versions of Figs. 5 and 7 are provided in this correction.
Publisher's Note Springer Nature remains neutral with regard to jurisdictional claims in published maps and institutional affiliations. 